\title{
Path Planning for the Mobile Robot: A Review
}

\author{
Han-ye Zhang * ${ }^{\mathbb{C}}$, Wei-ming Lin and Ai-xia Chen \\ School of Mechanical \& Materials Engineering, Jiujiang University, Jiujiang 332005, Jiangxi Province, China; \\ linwm123666@163.com (W.-m.L.); ChenAixia82@126.com (A.-x.C.) \\ * Correspondence: tzgjzhy@163.com
}

Received: 19 August 2018; Accepted: 22 September 2018; Published: 1 October 2018

\begin{abstract}
Good path planning technology of mobile robot can not only save a lot of time, but also reduce the wear and capital investment of mobile robot. Several methodologies have been proposed and reported in the literature for the path planning of mobile robot. Although these methodologies do not guarantee an optimal solution, they have been successfully applied in their works. The purpose of this paper is to review the modeling, optimization criteria and solution algorithms for the path planning of mobile robot. The survey shows GA (genetic algorithm), PSO (particle swarm optimization algorithm), APF (artificial potential field), and ACO (ant colony optimization algorithm) are the most used approaches to solve the path planning of mobile robot. Finally, future research is discussed which could provide reference for the path planning of mobile robot.
\end{abstract}

Keywords: path planning; mobile robot; environmental modeling; optimization criteria; path search

\section{Introduction}

Over the past decades, mobile robots have been successfully applied in different areas such as military, industry and security environments to execute crucial unmanned missions [1]. Path planning [2] is one of the most fundamental problems that have to be resolved before the mobile robots can navigate and explore autonomously in complex environments. Beginning with mid-1960s, the path planning has attracted interests from a lot of scholars. The path planning problem can be described in the following [3]: given a robot and its working environment, the mobile robots searches for an optimal or suboptimal path from the initial state to the target state according to a certain performance criteria. Good path planning technology of mobile robot can not only save a lot of time, but also reduce the wear and capital investment of mobile robot. Because the path planning of mobile robot has important application value, it has become a hot research topic both at home and abroad.

Generally speaking, the path planning can be divided into two categories: the global path planning and the local path planning (seen in Figure 1), according to whether all the information of the environment isaccessible or not. For the global path planning, all the information of the environment is known to the robot before starting. In contrast, for the local path planning, almost all the information of the environment is unknown to the robot before starting [4]. The path planning of mobile robot is retrieved by the database of Engineering Village, where the method of data retrieval is by Title: path planning \& mobile robot \& theme, for example, Title: path planning \& mobile robot \& genetic algorithm. Then, the results are summarized in Figure 2.

The remainder of the paper is as follows: Sections 2 and 3 provide the review of global path planning and local path planning, respectively. Section 4 concludes the paper. 


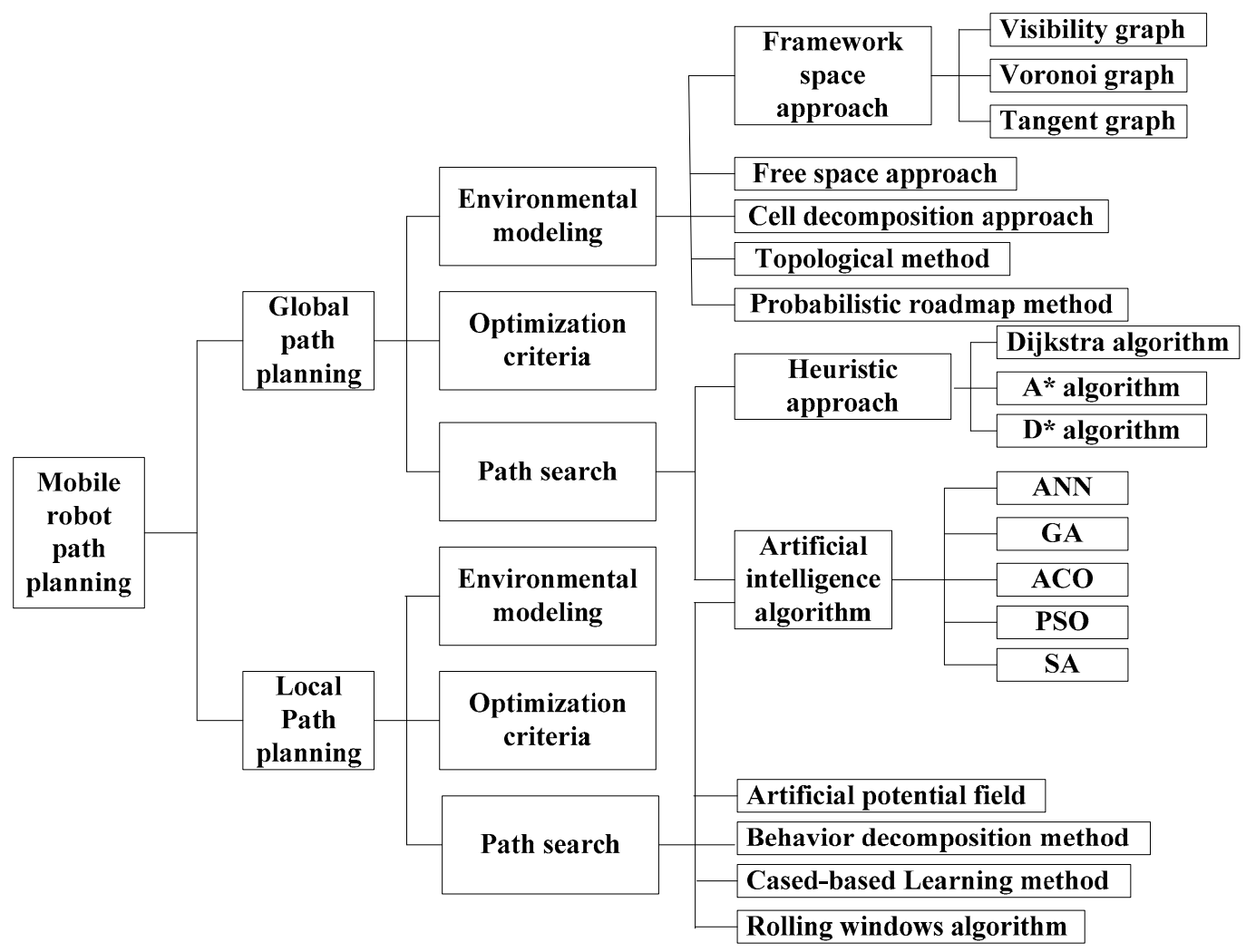

Figure 1. Classification of path planning.

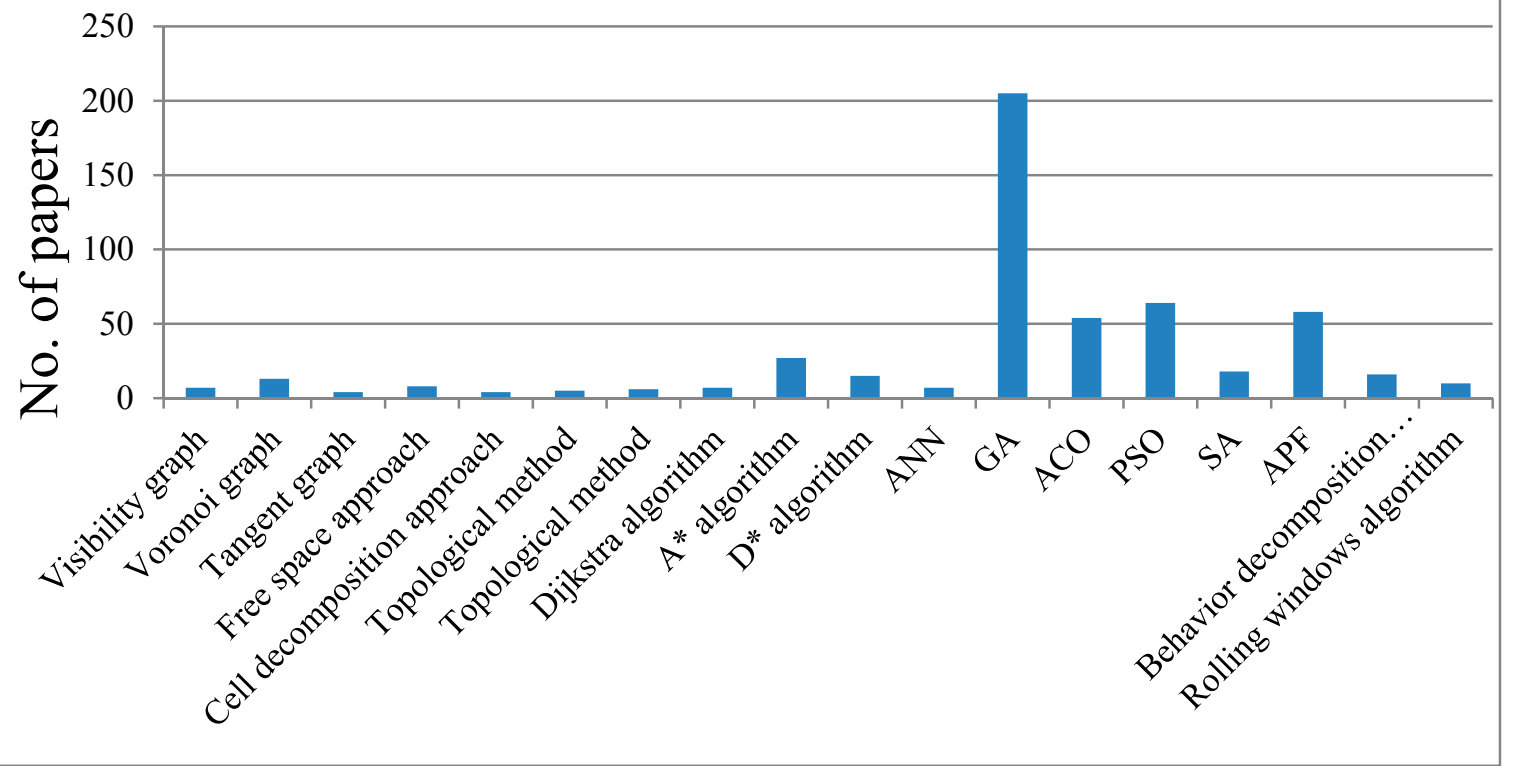

Figure 2. The number of papers retrieved by the database of Engineering Village. 


\section{Review of Global Path Planning}

In the process of global path planning of the mobile robot, the following steps should be followed in the general case. (1) Environmental modeling. The environmental modeling is built according to the known map information: the actual environment for the mobile robot to perform task is converted to the map feature information which can storage conveniently. (2) Optimization criteria. (3) Path search algorithm. The path search algorithm is adopted to find a collision free path between the starting point and the target point in the state space which must satisfy a set of optimization criteria such as path length, smoothness, safety degree, etc. The principle of mobile robot global path planning is shown in Figure 3.

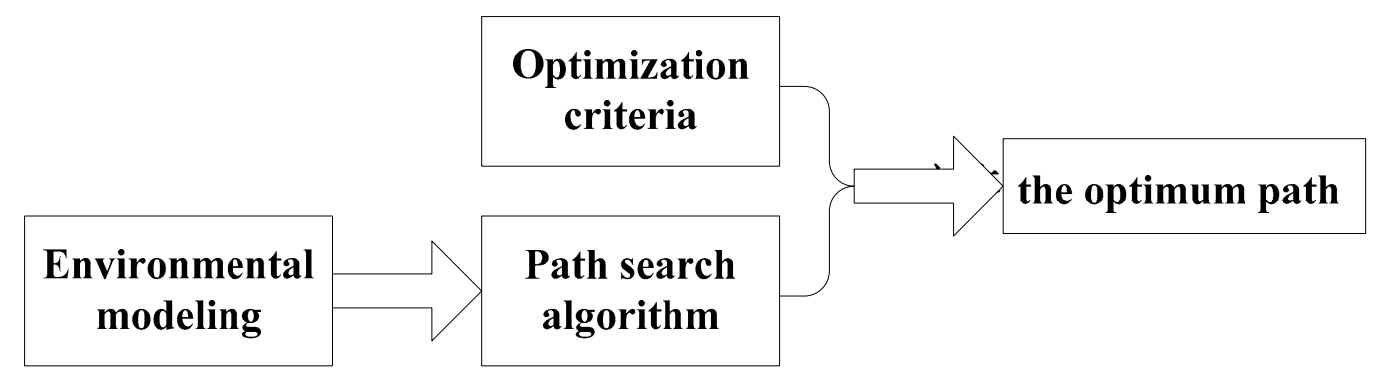

Figure 3. The principle of mobile robot global/local path planning.

\subsection{Environmental Modeling}

Before the mobile robot global path planning, a suitable environmental model will help to better understand the environmental variables, reduce unnecessary planning and greatly reduce the number of computations. Common methods of environmental modeling have framework space approach, free space approach, cell decomposition approach, topological method and probabilistic roadmap method.

\subsubsection{Framework Space Approach}

In order to simplify the problem, the mobile robot is usually reduced to a point, the obstacles around the mobile robot are scaled, the mobile robot can move freely in the obstacle space without colliding with obstacles and boundary. The framework space approach includes visibility graph, voronoi graph and tangent graph.

\section{Visibility Graph}

A polygon is used to represent an obstacle in the visibility graph (seen in Figure 4) method, and each endpoint is connected with all of its visible vertices to form a final map. In the range of the polygon, a vertex is connected to its total adjacent points, so the mobile robot can move along the polygon edge. Search the set of these lines and select an optimum path from the starting point to the end point. This method can successfully solve the small size problem in two-dimensional space and the path is optimal, but its time complexity is $O\left(N^{2}\right)$ [5]. However, with the increase of the problem's complexity, the efficiency of the visibility graph will be greatly reduced. If the visibility graph is used to the three-dimensional or a higher dimension space, then it is a NP-hard problem. At the same time, the mobile robot has a certain size and shape, all paths pass the end of obstacles, so the obtained path planning is likely to have a collision. 


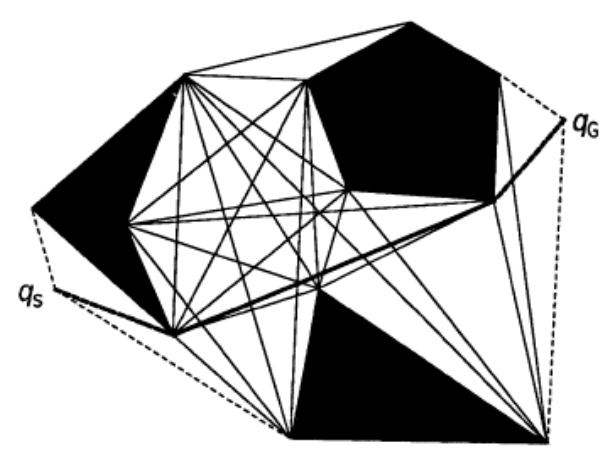

Figure 4. Visibility graph.

Voronoi Graph

The voronoi graph [6] (seen in Figure 5) is the trajectory of points that are equidistant from the nearest two or more barrier boundaries including the workspace boundary. The set of vertices is formed from points that are equidistant from three or more barrier boundaries, while the set of edges is formed from points that are equidistant from exactly two barrier boundaries. The merit of voronoi graph is of fast calculation speed and the drawback is of more mutational site.

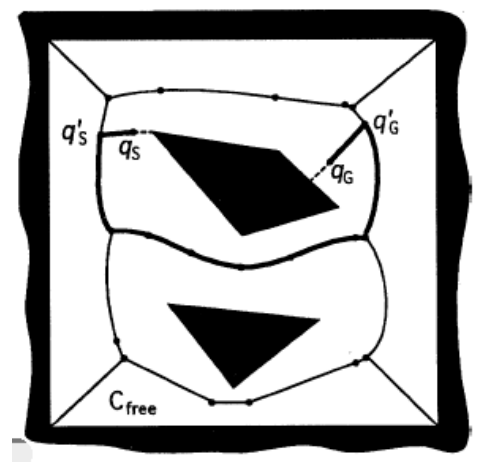

Figure 5. Voronoi graph.

\section{Tangent Graph}

In the tangent graph [7,8] (seen in Figure 6), the nodes represent tangent points on barrier boundaries, and the edges represent conflict-free common tangents of the obstacles or convex boundary segments between the tangent points. The tangent graph requires $O\left(K^{2}\right)$ memory, where $K$ represents the number of convex segments of the barrier boundaries. The disadvantage is that if the position error is generated in the control process, the possibility of robot collision obstacles will be very high.

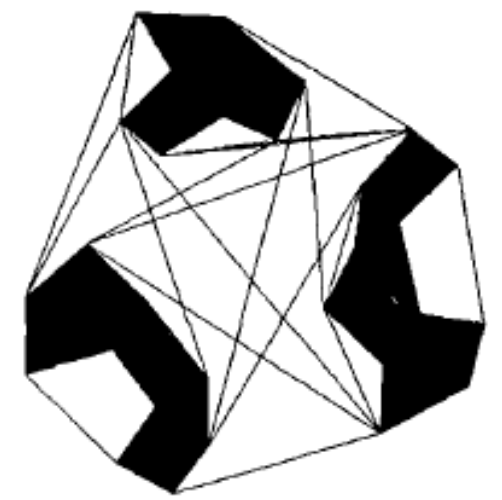

Figure 6. Tangent graph. 


\subsubsection{Free Space Approach}

Based on the concept of free link, Habib et al. [9] developed a new technique to construct the obtainable free space between obstacles within the robotic environment in terms of free convex region. Then, a new graph named MAKLINK is built to provide the generation of a conflict-free path. The graph is built by use of the midpoints of common free links between free convex region as the passing points. These points represent nodes, and the connection between the points within each convex region represent arcs in the graph. Aconflict-free path can be effectively generated by use of the MAKLINK graph. The complexity of searching for a conflict-free path is drastically reduced by minimizing the graphic size to be searched about the number of nodes and arcs connecting them. The advantage of free space approach is that it is more flexible, and easy to maintain the network diagram. In addition, it can flexibly change the starting point and target point of the robot. However, in an obstacle-intensive environment, the free space approach may fails and it can't obtain the optimal path.

\subsubsection{Cell Decomposition Approach}

The method decomposes the workspace of the mobile robot into a number of simple regions, and each region is generally called a cell. These grids form a connected graph and a path is searched from the initial grid to the target grid. In general, the path is represented by the ordinal number of the cell. The method is divided into two types: exact cell decomposition and approximate cell decomposition. The idea of exact cell decomposition is as follows. The free space is divided into $n$ non-overlapping units. The space after the combination of these $\mathrm{n}$ units is exactly the same as the original free space. In approximate cell decomposition, all of the grids are in a predetermined shape (e.g., rectangular). The whole environment is divided into a number of larger rectangles, each rectangle is continuous. If any big rectangle contains obstacles or boundary, then it is divided into 4 small rectangular, all the larger grid are executed this operation, the operation is repeated until it reaches the solution boundaries. This structure is called quadtree shown in Figure 7 [10].

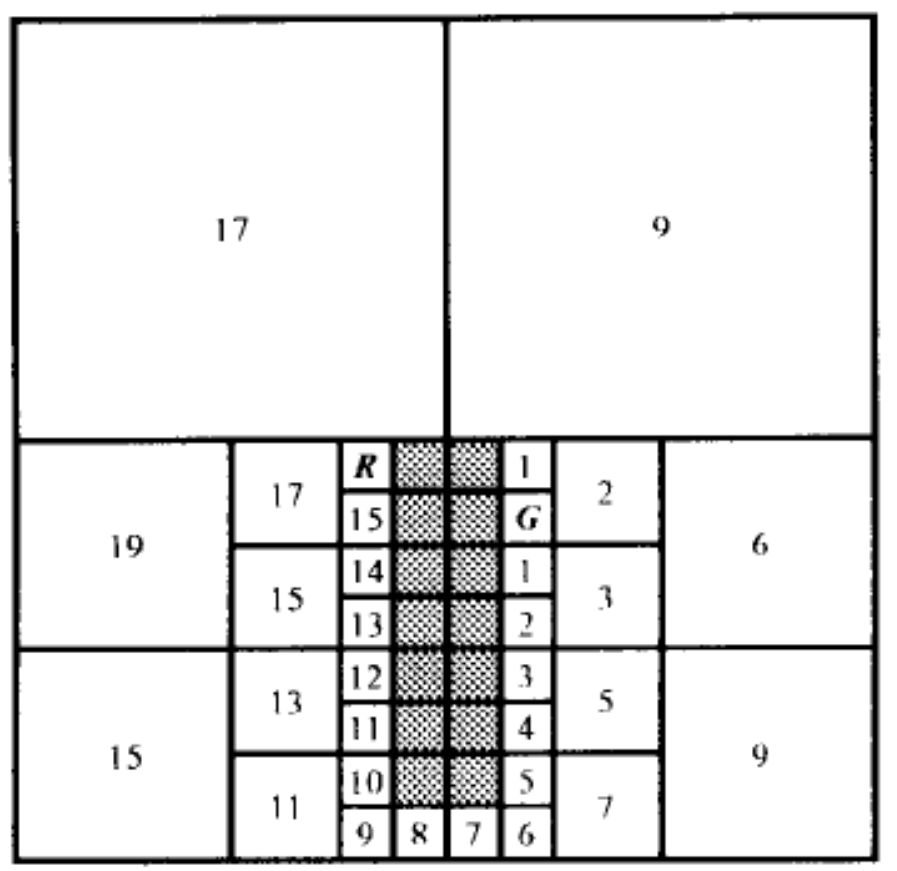

Figure 7. Standard quadtree-based approach. 


\subsubsection{Topological Method}

The topological method is method of reducing dimensions, and the path planning problem in high dimensional geometry space is transformed into the discriminant problem of connectivity in low dimension. When the topology network is established, the robot planning path is obtained from the starting point to the target point. Compared to the cell decomposition approach, this method only needs less model building time and less storage space, the complexity of the topological method only depends on the number of obstacles, the topological method can achieve fast path planning. Topology method is suitable for the environment with obvious characteristics and sparse obstacles; otherwise, it is difficult to carry out reliable navigation control. Another drawback is that topology method of environment information is not easy to maintain, when the number of obstacle is increased or decreased, the network is hard to modify, because the process of establishing the topology network itself is quite complex [11].

\subsubsection{Probabilistic Roadmap Method}

Kavraki et al. [12] proposed the probabilistic roadmap method in 1994. Some scholars continued to research it $[13,14]$. The main idea of probabilistic roadmap method is as follows. Based on random sample, an undirected roadmap graph $R=(N, E)$ is built, where $N$ is the nodes of obtained milestones by random sampling, $E$ is the edge connecting these nodes. Given the starting-point $s$ and finishing-point $f$, the probabilistic roadmap method is looking for two nodes $s^{\prime}$ and $f^{\prime}$ satisfying $s$ and $s^{\prime}$ are directly connected, $f$ and $f^{\prime}$ are directly connected. A path planning is obtained by searching the edge sequence which are directly connected to $s^{\prime}$ and $f^{\prime}$ in the undirected roadmap graph.

\subsection{Optimization Criteria}

Generally speaking, there are many factors that must be considered in the optimization criteria for planning a mobile path. Three commonly used optimization criteria are listed in the following.

\subsubsection{Path Length}

The path length $D[15,16]$ is defined as

$$
D=\sum_{j=0}^{n-1} \sqrt{\left(x_{j+1}-x_{j}\right)^{2}+\left(y_{j+1}-y_{j}\right)^{2}}
$$

where, $x_{j}$ and $y_{j}$ are the values of the $X$ coordinate axis and $Y$ coordinate axis of the nodes $j$, respectively.

\subsubsection{Smoothness}

The smoothness $S$ [15] is defined as

$$
S=\alpha \cdot\left(1-\frac{D A_{l}}{N_{f}-1}\right)+\beta \cdot\left(1-\frac{S_{\min }}{N_{f}}\right)
$$

where, $\alpha$ and $\beta$ are weighted coefficients, $D A_{l}$ is the number of angle of deflection larger than the desired variable, $N_{f}$ is the total number of path segments, $S_{\min }$ is the number of segments with the smallest number of path segments in the path.

\subsubsection{Safety Degree}

The safety degree $S D[15]$ is defined as

$$
S D=\sum_{j=1}^{n-1} C_{j}=\left\{\begin{array}{c}
0, d_{j} \geq \lambda \\
\sum_{j=1}^{n-1} e^{\lambda-d_{j}}, d_{j}<\lambda
\end{array}\right.
$$


where, $d_{i}$ is the minimal distance between the $i$-th segment and its nearest obstacle, and $\lambda$ is the threshold of the safety degree.

\subsection{Path Search Algorithm}

Generally speaking, the path search algorithm for the global path planning can beclassified into two categories: heuristic approach and artificial intelligence algorithm.

\subsubsection{Heuristic Approach}

\section{Dijkstra Algorithm}

The Dijkstra algorithm is proposed by E.W. Dijkstra in 1959 [17]. It is a typical shortest path algorithm for solving the shortest path problem in a directed graph. Its main feature is that the starting point is as the center to be extended to the end point.

Each edge of the graph is formed to an ordered element pair by the two vertices. The value of the edges are described by the weight function. The algorithm maintains two vertex sets named $A$ and $B$. The initial set $A$ is empty. Each time a vertex in $B$ is moved to $A$, and the selected vertex ensures the sum of all the edge weight from the starting point to the point is minimized. Because the algorithm needs to traverse more nodes, so the efficiency is not high.

\section{$A^{*}$ Algorithm}

Hart et al. [18] proposed $A^{*}$ algorithm in 1968.

The $A^{*}$ algorithm is developed on the basis of the Dijkstra algorithm. Starting from a specific node, the weighted value of the current child nodes are updated, and the child node which has the smallest weighted value is used to update the current node until all nodes are traversed. The key of $A^{*}$ algorithm is to establish the evaluation function $f(n), f(n)=g(n)+h(n)$, where $g(n)$ represents the actual cost from the initial node to the node $n$, and $h(n)$ represents the estimated cost of the optimal path from node $n$ to the target node in the state space. The Euclidean distance between the two nodes is usually taken as the value of $h(n)$. When the value of $g(n)$ is constant, the value of $f(n)$ is mainly affected by the value of $h(n)$. When the node is close to the target node, the value of $h(n)$ is small, the value of $f(n)$ is relatively small. As a result, it guarantees the search for the shortest path always proceeds in the direction of the target point. The $A^{*}$ algorithm considers the position information of the mobile robot's target point and searches along the target point. Compared with the Dijkstra algorithm, the path search efficiency of the $A^{*}$ algorithm is higher.

\section{$D^{*}$ Algorithm}

$A^{*}$ algorithm is mainly used for the global search of the static environment. However, the path planning of mobile robots in practical application is gradually aware of the environmental information, and it is dynamic. Stentz [19] proposed $D^{*}$ algorithm in 1994. It is mainly used for robot to explore the path. The problem space of the $D^{*}$ algorithm is expressed as a series of state, and the states represent the direction of the robot's position. The principle of $D^{*}$ algorithm is basically the same as that of $D^{*}$ algorithm, the cost of arc used to ensure the direction of the search. In addition, some scholars have researched the $D^{*}$ algorithm such as the field $D^{*}$ algorithm [20] and Theta* algorithm [21,22].

\subsubsection{Artificial Intelligence Algorithm}

\section{ANN}

Path planning is a kind of mapping from the perceptual space to the space of behavior, and the artificial neural network (ANN) can express the mapping relationship.

The neural network is used to describe the constraints among the environment, and the energy is defined as the function of the path point. The level of the energy depends on the location of the path 
point, and the robot moves towards the direction of diminished energy. A path with the smallest total energy is obtained at last. Although this path has no obstacles, it is not the shortest or optimal path. Martin et al. [23] used ANN to solve the robotic path planning problem and discussed how neural networks may contribute to increase the performance of robotic path planners. Mulder et al. [24] constructed an interactive and competitive ANN tosettle the path planning problems. Combined ANN and Q-learning, Li et al. [25] proposed a hybrid method for solving the robotic path planning. The results show the hybrid method was better than either of the two methods. Raza et al. [26] used evolutionary ANN to solve path planning in RoboCup soccer. Contreras-Gonzalez et al. [27] proposed a back-propagation ANN for solving the path planning. The working environment of the mobile robot is random and it is difficult to describe by mathematical formula. It is hard to establish a neural network topology to describe the moving environment. In addition, the complex and large structure makes the weight setting of the neural network to be difficult.

\section{GA}

The genetic algorithm (GA) is proposed by Holland in 1975. In the GA, all the possible solutions of the problem are encoded to chromosomes, and all the chromosomes form an initial population. Several basic operations are constructed: crossover, mutation and selection. Initial population is generated, then the fitness value of each individual is calculated by the objectives. The individuals which are selected for crossover operation, mutation operation and selection operation are determined by the fitness value. The flowchart of GA is shown in Figure 8. Min et al. [28] used GA to settle the path planning for mobile manipulator. Liu et al. [29] presented a GA with two-layer encoding to settle the path planning. This kind of encoding can improve the expressing ability of codes. The heart of the two-layer encoding is to decrease the complexity of exploration through the middle-layer codes. Pehlivanoglu et al. [30] proposed vibrational genetic algorithm for path planning. Xu et al. [31] presented adaptive GA to solve the path planning of unmanned aerial vehicle. Example simulation shows that the new algorithm satisfies the requirements in the computation efficiency and the precision of the solution. Tsai et al. [32] proposed PEGA (parallel elite genetic algorithm) for autonomous robot navigation. The results show the PEGA is effective. Tuncer et al. [33] proposed an improved GA for mobile robots' dynamic path planning. Qu et al. [34] proposed an improved GA with co-evolutionary strategy to solve the global path planning for multiple mobile robots. The simulations show the method is efficient. Fei et al. [35] proposed tailored GA for mobile robot's optimal path planning. Shorakaei et al. [36] used a parallel GA for unmanned aerial vehicles' optimal cooperative path planning. The effectiveness of the method was shown by several simulations. The advantage of GA is that it is simple, robust, and has strong search capability and high search efficiency. However, it is prone to premature convergence. When it approaches the optimal solution of the problem, the convergence speed of the algorithm will decrease. It is usually used in the global path planning. 


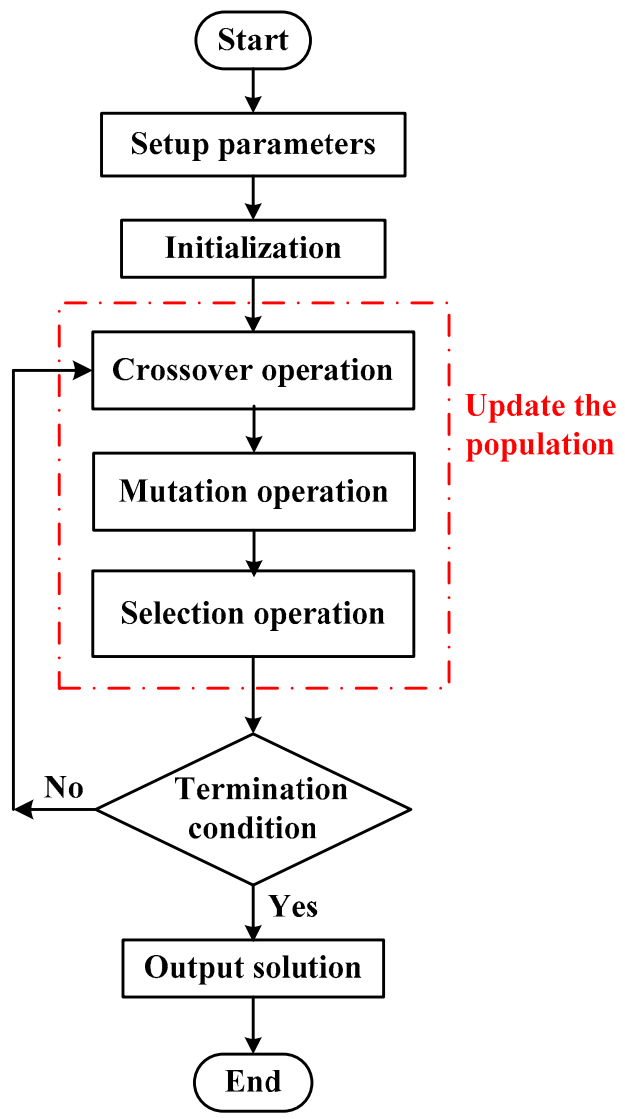

Figure 8. The flowchart of GA.

$\mathrm{ACO}$

The ACO (Ant colony optimization algorithm) is proposed by Marco Dorigo in 1992.

The basic principle of the ACO is each ant will release a secretion on the path it walked as a reference and will also perceive the secretions released by other ants while it is searching for food. This secretion is usually called pheromone. Under the action of pheromones, the ant colony can communicate with each other and choose paths. When the pheromone on a path is more than other paths, the ant colony will spontaneously move to this path, and release more secretions during the movement, so that the concentration of the pheromone becomes higher to attract the latter ants which forms a mechanism of positive feedback. After a period of time, the concentration of pheromone on the shorter path is getting higher and higher, then the ants that choose it are gradually increasing, while the pheromones on other paths are gradually reduced until there is no. Finally the whole ant colony is concentrated in the optimal path. The process of ant foraging is similar to the path planning of robots. As long as there are enough ants in the nest, these ants will find the shortest path from the nest to the food to avoid obstacles. The principle of ant colony searching for food is shown in Figure 9. Wen et al. [37] modified ACO to optimize the global path. When only the pheromone was used to search the optimum path, the ACO converges easily. Wang et al. [38] used ACO to research on global path planning. Simulation results show the ACO algorithm is suitable for global path planning. Zhu et al. [39] proposed an improved ACO for the path planning of mobile robot. The results show the algorithm can not only increase the performance of path planning, but also the algorithm is effective. Zhao et al. [40] improved ACO to solve path planning of mobile robot. Simulation results show the improved algorithm converges quickly even in complex environment. Gao et al. [41] proposed an improved ACO for mobile robot's three-dimensional path planning. The results show that it was an effective approach. You et al. [42] proposed a chaotic ant colony system to solve the path planning of mobile robot. Simulation results show that the approach is not only more effective than the traditional 
ant colony system, but also improves the global search capabilities. The ACO has not only the global search ability of the population, but also has synergy between individuals. It can find a better path, even if the complete information of the environment is not known. However, in the early stage of the algorithm, the convergence speed is slow and it takes a lot of computation time. It is prone to prematurity.
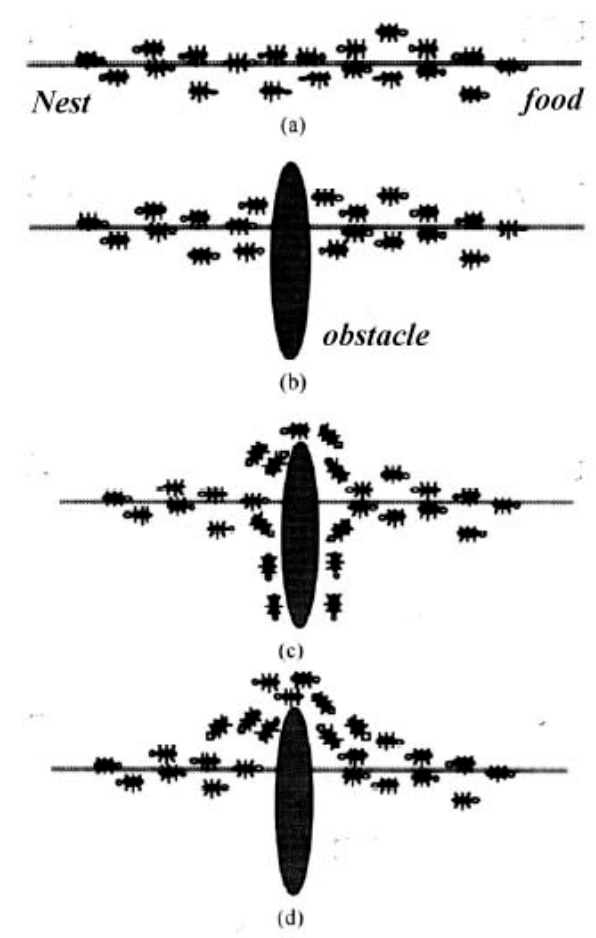

Figure 9. The principle of ant colony searching for food (ant colony searching for food from (a) to (d)). PSO

Inspired by the regularity of the bird cluster activity, Eberhart and Kennedy proposed the PSO (particle swarm optimization) algorithm in 1995. It starts from a random solution. It finds the optimal solution through iteration. It evaluates the quality of the solution through fitness value, and it finds the global optimal by comparing the currently searched optimal value at last. This algorithm is used to solve the robotic path planning with the advantages of easy implementation, high precision and fast convergence. Zhang et al. [43] presented an improved PSO for a mobile robot's path planning. Simulation results show the method is effective. Based on multi objective PSO, Gong et al. [44] proposed a global path planning method. The effectiveness of the algorithm is verified by simulation. An improved chaos PSO was proposed to solve the path planning for unmanned aerial vehicle [45]. The results show the proposed algorithm was superior to the traditional PSO, especially in the three-dimensional environment. A fitness-scaling adaptive Chaotic PSO approach was presented to solve the path planning of UCAVs [46]. Based on PSO, Liu et al. [47] introduced some key technologies for path planning in radiation environment. The probability and effectiveness of the method is verified by the experiment. Yusof et al. [48] proposed a predetermined waypoints method. The results show the approach is promising. The algorithm is fast and efficient, but it is easy to fall into local optimum.

SA

The idea of simulated annealing (SA) algorithm was proposed by N. Metropolis et al. in 1953. It is a stochastic optimization algorithm based on the iterative solution strategy of Monte-Carlo. The SA starts from a certain higher initial temperature, and with the continuous decrease of temperature 
parameters, it randomly finds the global optimal solution of the objective function in the solution space combined with the feature of probability jump.

Martinez-Alfaro et al. [49] used SA to obtain an optimal conflict-free path for mobile robots or AGV in two-dimensional and three-dimensional environment. Vougioukas et al. [50] proposed an accelerated SA algorithm to resolve the path planning. Miao et al. [51] proposed a SA approach to obtain the optimal or near-optimal path quickly for a mobile robot in dynamic environments with static and dynamic obstacles. The effectiveness of the proposed approach was demonstrated. Chiu [52] used the SA to solve the path planning problem for mobile robots. The results show the method is effective. Hui et al. [53] developed an enhanced SA approach to solve the dynamic robot path planning. Behnck et al. [54] developed a modified SA algorithm to solve the path planning for SUAVs. The results show the modified SA is able to calculate paths matching POI and UAV types with an execution time. The algorithm has slow convergence speed, long execution time, and the performance relies on the initial value.

\section{Review of Local Path Planning}

The contents of environmental modeling and optimization criteria refer to the previous section of review of global path planning.

The path search algorithm for the local path planning can be divided into five categories: artificial potential field method, behavior decomposition method, cased-based Learning method, rolling windows algorithm, and artificial intelligence algorithm.

\subsection{Artificial Potential Field}

The idea of artificial potential field (APF) comes from the concept of potential field in physics, which regards the movement of objects as the result of two kinds of forces. The robot in the planning space is subjected to the gravitational force from the target point and repulsed by the obstacle. Under the action of the two forces, the robot moves toward the target point in the resultant force, and during the movement process it can effectively avoid the obstacles in the planning space and reach the target safely. The scheme of artificial potential field is shown in Figure 10.

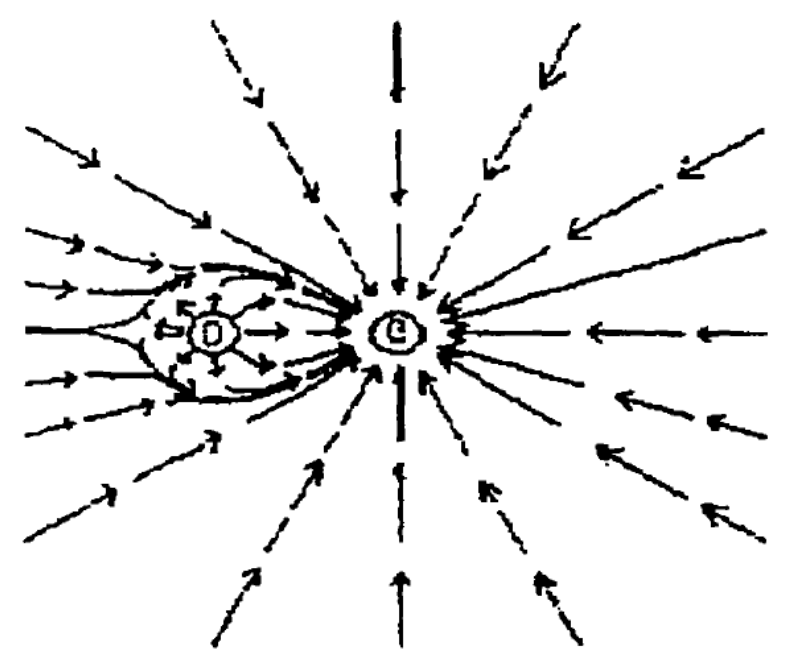

Figure 10. The scheme of artificial potential field.

Vadakkepat et al. [55] proposed a new approach called evolutionary APF (EAPF) for path planning of the real-time robot. The robustness and efficiency are verified by simulation. Min GP et al. [56] presented a virtual obstacle concept base on the APF to study the path planning of mobile robot. The results show the method is feasible and of small complexity. Cao et al. [57] proposed a modified APF approach for the path planning of mobile robot in a dynamic environment. Computer simulation 
and experiment demonstrated the effectiveness of the dynamic path-planning scheme. In order to solve the problems that path planning trapped in local minimum, Zhang et al. [58] proposed the evolutionary APF method. The feasibility and effectivity are verified by simulation. Zhou et al. [59] proposed an adaptive APF method for the path planning of robot obstacle avoidance. The results show the method can avoid falling into the local optimal solution. The merits of APF: (1) it is easy to operate and realize; (2) it can get a more secure path; (3) it needs a little map information and does not need a lot of computing in the planning process; (4) it can get a smoother trajectory. However, the APF ha some shortcomings, such as: (1) it is prone to shock before the obstacles; (2) if there is an obstacle near the target, the robot moves toward the target point, the smaller the distance between the robot and the target point, the greater the repulsion which will lead such a result the robot fail to reach the target point; (3) the path planning will trap into a locally optimal solution in some areas, etc.

\subsection{Behavior Decomposition Method}

Behavior decomposition method is a new trend in the path planning of mobile robot. Briefly speaking, it is to break down the navigation problem into a number of relatively independent navigation unit: behavioral primitives, such as collision avoidance, tracking, target guidance, etc. These behavior units are complete motion control unit with sensors and actuators, and have corresponding navigation. These behavior units coordinate with each other to complete the overall navigation tasks. Whitbrook et al. [60] integrated an idiotypic artificial immune system network with a reinforcement-learning-based control system for behavior planning control in robot navigation. Huq et al. [61] proposed a new approach combined fuzzy context dependent behavior modulation and motor schema. The results show it can obtain a conflict-free goal. Fernandez-Leon et al. [62] study the behaviors of scaling up in evolutive robotics. The results show it is efficient. Combined the improved beam curvature method (BCM) and the prediction model of collision, Shi et al. [63] presented a new method of local obstacle avoidance. The results show it can avoid moving obstacles in the dynamic environments. Toibero et al. [64] presented a switching control approach for the parking problem of non-holonomic mobile robot. The results show it is feasible.

\subsection{Cased-Based Learning Method}

Mobile robot needs to establish a proper case database before path planning. When the mobile robot encounters a new problem, it will search the information from the established case database. Based on the search results, it will compare and analyze to find a solution that is most similar to the new problem. Marefat et al. [65] developed a process planning system. The efficiency and the effectiveness of the approach is verified by experiment. Experience knowledge is accumulated from training and match practice, and is mainly used to match, as most knowledge is derived from experience. The way and formula are discussed and they succeed in applying to robotics soccer [66]. Case-based learning method was applied to the motion planning where volleyball robot's initial state was partially changed [67]. An intelligent typical case-based reasoning to path planning was put forward [68]. Combined with the knowledge about the road network, typical cases were defined and used to solve the problem. The experimental result showed that this path-planning algorithm can reduce the search space, speed up the progress of searching and satisfy people's preferences of running on the road they are familiar with.

\subsection{Rolling Windows Algorithm}

The mobile robot path planning based on the rolling window method uses the local environment information obtained by the mobile robot to establish a "window", and the path planning is realized by recursively calculating the "window" with its own surrounding information. At each step of the rolling calculation, sub-targets are obtained by heuristic method, then the obtained sub-targets are implemented real-time planning in the current rolling window. With the moving of the rolling window, the sub-targets are updated by the obtained information until the planning task is completed. 
Combined rolling path planning and bug algorithm, Zhong et al. [69] presented a new approach to solve the path planning of mobile robot. The approach has environmental adaptability and good capability of obstacle avoidance by simulation. Combining the advantages of least-squares policy iteration (LSPI) and path planning based on rolling windows, a novel reactive navigation method based on LSPI, and rolling windows was presented [70]. The effectiveness and the adaptiveness are verified by simulation and experiment. Based on the dynamic window approach (DWA) for robot navigation, Chou et al. [71] proposed an approach named DWA*. The results show this approach has high performance by simulation and experiment. Because the local environment information of the mobile robot is measured in real time and is online planned in a rolling manner, it has good collision avoidance capability. However, the method may be trapped in a local optimization, it does not guarantee the obtained path is the optimal solution.

\subsection{Artificial Intelligence Algorithm}

The contents of artificial intelligence algorithm refers to the previous section of review of global path planning.

\section{Conclusions}

The path planning problem is an important research field of the mobile robot which has aroused the interest of many researchers both at home and abroad. Good path planning technology of mobile robot can not only save a lot of time, but also reduce the wear and capital investment of mobile robot. Different methodologies have been reviewed in this paper. The results shows GA, PSO, APF, and ACO are the most used four approaches to solve the path planning of mobile robot. Finally, future research is discussed which could provide reference for the path planning of mobile robot. Future research should include: (1) Each method can be suitable for different applications. As yet, there is no universal algorithm or method that can solve all above cases. The new path planning method should be researched, such as artificial immune algorithm [72], artificial bee colony [73,74], etc. Especially two or more algorithms are combined to improve the quality and efficiency of the solution. (2) Multi-sensor information should be inosculated into the path planning. Multi-sensor information fusion technology can overcome uncertainty and information incompleteness of the single sensor. It can more accurately and comprehensively understand and describe the environment and the measured object. (3) The task assignment, communication cooperation and path planning of multi-robot should be researched. (4) Path planning of mobile robots in high dimensional environment should be researched. (5) Air robot and underwater robot should be researched. (6) The combination of the robot bottom control and path planning algorithm should be researched.

Author Contributions: H.-y.Z. wrote the original manuscript; W.-m.L. and A.-x.C. polished the manuscript.

Funding: This research received no external funding.

Conflicts of Interest: The authors declare no conflicts of interest.

\section{References}

1. Tang, B.W.; Zhu, Z.X.; Luo, J.J. Hybridizing Particle Swarm Optimization and Differential Evolution for the Mobile Robot Global Path Planning. Int. J. Adv. Robot. Syst. 2016, 13, 1-17. [CrossRef]

2. Zhuang, Y.; Sun, Y.L.; Wang, W. Mobile robot hybrid path planning in an obstacle-cluttered environment based on steering control and improved distance propagating. Int. J. Innov. Comput. Inf. Control 2012, 8, 4095-4109.

3. Contreras-Cruz, M.A.; Ayala-Ramirez, V.; Hernandez-Belmonte, U.H. Mobile robot path planning using artificial bee colony and evolutionary programming. Appl. Soft Comput. 2015, 30, 319-328. [CrossRef]

4. Li, P.; Huang, X.H.; Wang, M. A novel hybrid method for mobile robot path planning in unknown dynamic environment based on hybrid DSm model grid map. J. Exp. Theor. Artif. Intell. 2011, 23, 5-22. [CrossRef] 
5. Goerzen, C.; Kong, Z.; Mettler, B. A survey of motion planning algorithms from the perspective of autonomous UAV guidance. J. Intell. Robot. Syst. Theory Appl. 2010, 57, 65-100. [CrossRef]

6. Hoang, H.V.; Sang, H.A.; Tae, C.C. Dyna-Q-based vector direction for path planning problem of autonomous mobile robots in unknown environments. Adv. Robot. 2013, 27, 159-173.

7. Liu, Y.; Arimoto, S. Proposal of tangent graph and extended tangent graph for path planning of mobile robots. In Proceedings of the 1991 IEEE International Conference on Robotics and Automation, Sacramento, CA, USA, 9-11 April 1991; pp. 312-317.

8. Henrich, D. Fast motion planning by parallel processing-A review. J. Intell. Robot. Syst. Theory Appl. 1997, 20, 45-69. [CrossRef]

9. Habib, M.K.; Asama, H. Efficient method to generate collision free paths for an autonomous mobile robot based on new free space structuring approach. In Proceedings of the IEEE/RSJ International Workshop on Intelligent Robots and Systems (IROS'91), Osaka, Japan, 3-5 November 1991; pp. 563-567.

10. Chen, D.Z.; Szczerba, R.J.; Uhran, J.J. Framed-quadtree approach for determining Euclidean shortest paths in a 2-D. environment. IEEE Trans. Robot. Autom. 1997, 13, 668-681. [CrossRef]

11. Liang, W.J. Research on Robot Path Planning for Dynamic Environment and Cooperation. Master's Thesis, Zhejiang University, Hangzhou, China, 2010.

12. Kavraki, L.; Latombe, J. Randomized preprocessing of configuration space for fast path planning. In Proceedings of the 1994 IEEE International Conference on Robotics and Automation, San Diego, CA, USA, 8-13 May 1994; pp. 2138-2145.

13. Kavraki, L.E.; Svestka, P.; Latombe, J.; Overmars, M.H. Probabilistic roadmaps for path planning in high-dimensional configuration spaces. IEEE Trans. Robot. Autom. 1996, 12, 566-580. [CrossRef]

14. Hsu, D.; Latombe, J.; Kurniawati, H. On the probabilistic foundations of probabilistic roadmap planning. Int. J. Robot. Res. 2006, 25, 627-643. [CrossRef]

15. Wang, X.Y.; Zhang, G.X.; Zhao, J.B.; Rong, H.; Ipate, F.; Lefticaru, R. A Modified Membrane-Inspired Algorithm Based on Particle Swarm Optimization for Mobile Robot Path Planning. Int. J. Comput. Commun. Control 2015, 10, 732-745. [CrossRef]

16. Hidalgo-Paniagua, A.; Vega-Rodriguez, M.A.; Ferruz, J.; Pavón, N. MOSFLA-MRPP, Multi-Objective Shuffled Frog-Leaping Algorithm applied to Mobile Robot Path Planning. Eng. Appl. Artif. Intell. 2015, 44, 123-136. [CrossRef]

17. Dijkstra, E. A note on two problems in connexion with graphs. Numer. Math. 1959, 4, 269-271. [CrossRef]

18. Hart, P.E.; Nilsson, N.J.; Raphael, B. A formal basis for the heuristic determination of minimum cost paths in graphs. IEEE Trans. Syst. Sci. Cybern. 1968, 4, 100-107. [CrossRef]

19. Stentz, A. Optimal and efficient path planning for partially-known environments. In Proceedings of the 1994 IEEE International Conference on Robotics and Automation, San Diego, CA, USA, 8-13 May 1994; pp. 3310-3317.

20. Ferguson, D.; Stentz, A. Using interpolation to improve path planning the field D*algorithm. J. Field Robot. 2006, 23, 79-101. [CrossRef]

21. Nash, A.; Daniel, K.; Koenig, S.; Felner, A. Theta*, Any-angle path planning on grids. J. Artif. Intell. Res. 2010, 39, 533-579.

22. Xiao, K.; Gao, C.; Hu, X.; Pan, H.W. Improved Theta*, Improved any-angle path planning on girds. J. Comput. Inf. Syst. 2014, 10, 8881-8890.

23. Martin, P.; Del Pobil, A.P. Application of artificial neural networks to the robot path planning problem. In Proceedings of the Ninth International Conference on Applications of Artificial Intelligence in Engineering, Pennsylvania, PA, USA, 19-21 July 1994; pp. 73-80.

24. Mulder, E.; Mastebroek, H.A.K. Construction of an interactive and competitive artificial neural network for the solution of path planning problems. In Proceedings of the European Symposium on Artificial Neural Networks, Bruges, Belgium, 22-24 April 1998; pp. 407-412.

25. Li, C.H.; Zhang, J.Y.; Li, Y.B. Application of artificial neural network based on Q-learning for mobile robot path planning. In Proceedings of the 2006 IEEE International Conference on Information Acquisition, Weihai, China, 20-23 August 2006; pp. 978-982.

26. Raza, S.; Haider, S. Path planning in RoboCup soccer simulation 3D using evolutionary artificial neural network. In Proceedings of the Advances in Swarm Intelligence 4th International Conference, Harbin, China, 12-15 June 2013; pp. 342-350. 
27. Contreras-Gonzalez, A.; Hernandez-Vega, J.; Hernandez-Santos, C.; Palomares-Gorham, D.G. A method to verify a path planning by a back-propagation artificial neural network. In Proceedings of the 10th Latin American Workshop on Logic/Languages, Algorithms and New Methods of Reasoning, Puebla, Mexico, 15 August 2016; pp. 98-105.

28. Min, Z.; Ansari, N.; Hou, E.S.H. Mobile manipulator path planning by a genetic algorithm. J. Robot. Syst. 1994, 11, 143-153.

29. Liu, Y.F.; Qiu, Y.H. Robot path planning based on genetic algorithms with two-layer encoding. Control Theory Appl. 2000, 17, 429-432.

30. Pehlivanoglu, Y.V.; Baysal, O.; Hacioglu, A. Path planning for autonomous UAV via vibrational genetic algorithm. Aircr. Eng. Aerosp. Technol. 2007, 79, 352-359. [CrossRef]

31. Xu, Z.; Tang, S. UAV path planning based on adaptive genetic algorithm. J. Syst. Simul. 2008, 20, 5411-5414.

32. Tsai, C.; Huang, H.; Chan, C. Parallel elite genetic algorithm and its application to global path planning for autonomous robot navigation. IEEE Trans. Ind. Electron. 2011, 58, 4813-4821. [CrossRef]

33. Tuncer, A.; Yildirim, M. Dynamic path planning of mobile robots with improved genetic algorithm. Comput. Electr. Eng. 2012, 38, 1564-1572. [CrossRef]

34. Qu, H.; Xing, K.; Alexander, T. An improved genetic algorithm with co-evolutionary strategy for global path planning of multiple mobile robots. Neurocomputing 2013, 120, 509-517. [CrossRef]

35. Liu, F.; Liang, S.; Xian, X.D. Optimal Path Planning for Mobile Robot Using Tailored Genetic Algorithm. IAES TELKOMNIKA Indones. J. Electr. Eng. 2014, 12, 1-9. [CrossRef]

36. Shorakaei, H.; Vahdani, M.; Imani, B.; Gholami, A. Optimal cooperative path planning of unmanned aerial vehicles by a parallel genetic algorithm. Robotica 2016, 34, 823-836. [CrossRef]

37. Wen, Z.Q.; Cai, Z.X. Global path planning approach based on ant colony optimization algorithm. J. Cent. South Univ. Technol. 2006, 13, 707-712. [CrossRef]

38. Wang, H.J.; Xiong, W. Research on global path planning based on ant colony optimization for AUV. J. Mar. Sci. Appl. 2009, 8, 58-64. [CrossRef]

39. Zhu, X.; Han, Q.; Wang, Z. The application of an improved ant colony algorithm in mobile robot path planning. Key Eng. Mater. 2011, 467-469, 222-225. [CrossRef]

40. Zhao, J.; Fu, X. Improved ant colony optimization algorithm and its application on path planning of mobile robot. J. Comput. 2012, 7, 2055-2062. [CrossRef]

41. Gao, M.K.; Chen, Y.M.; Liu, Q.; Huang, C.; Li, Z.; Zhang, D. Three-dimensional path planning and guidance of leg vascular based on improved ant colony algorithm in augmented reality. J. Med. Syst. 2015, 39, 111-133. [CrossRef] [PubMed]

42. You, X.; Liu, K.; Liu, S. A chaotic ant colony system for path planning of mobile robot. Int. J. Hybrid Inf. Technol. 2016, 9, 329-338. [CrossRef]

43. Zhang, Q.R.; Li, S.H. Path planning based on improved particle swarm optimization for a mobile robot. WSEAS Trans. Syst. Control 2007, 2, 347-352.

44. Gong, D.W.; Zhang, J.H.; Zhang, Y. Multi-objective Particle Swarm Optimization for Robot Path Planning in Environment with Danger Sources. J. Comput. 2011, 6, 1554-1561. [CrossRef]

45. Cheng, Z.; Tang, Y.X.; Liu, Y.L. 3-D Path Planning for UAV Based on Chaos Particle Swarm Optimization. Appl. Mech. Mater. 2012, 232, 625-630. [CrossRef]

46. Zhang, Y.D.; Wu, L.N.; Wang, S.H. UCAV Path Planning by Fitness-scaling Adaptive Chaotic Particle Swarm Optimization. Math. Probl. Eng. 2013, 2013, 705238. [CrossRef]

47. Liu, Y.K.; Li, M.K.; Xie, C.L.; Peng, M.; Xie, F. Path-planning research in radioactive environment based on particle swarm algorithm. Prog. Nucl. Energy 2014, 74, 184-192. [CrossRef]

48. Yusof, T.S.T.; Toha, S.F.; Yusof, H. Path Planning for Visually Impaired People in an Unfamiliar Environment Using Particle Swarm Optimization. Procedia Comput. Sci. 2015, 76, 80-86. [CrossRef]

49. Martinez-Alfaro, H.; Flugrad, D.R. Collision-free path planning for mobile robots and/or AGVs using simulated annealing. In Proceedings of the 1994 IEEE International Conference on Systems, Man and Cybernetics, San Antonio, TX, USA, 2-5 October 1994.

50. Vougioukas, S.; Ippolito, M.; Cugini, U. Path planning based on accelerated simulated annealing. In Proceedings of the 1996 Research Workshop on ERNET-European Robotics Network, Darmstadt, Germany, 9-10 September 1996; pp. 259-268. 
51. Miao, H.; Tian, Y.C. Robot path planning in dynamic environments using a simulated annealing based approach. In Proceedings of the 2008 10th International Conference on Control, Automation, Robotics and Vision, Hanoi, Vietnam, 17-20 December 2008; pp. 1253-1258.

52. Chou, M.C. Path-planning for an autonomous robot using a simulating annealing. J. Inf. Optim. Sci. 2011, 32, 297-314. [CrossRef]

53. Miao, H.; Tian, Y.C. Dynamic robot path planning using an enhanced simulated annealing approach. Appl. Math. Comput. 2013, 222, 420-437. [CrossRef]

54. Behnck, L.P.; Doering, D.; Pereira, C.E.; Rettberg, A. A modified simulated annealing algorithm for SUAVs path planning. IFAC-PapersOnLine 2015, 48, 63-68. [CrossRef]

55. Vadakkepat, P.; Tan, K.C.; Wang, M.L. Evolutionary Artificial Potential Fields and their application in real time robot path planning. In Proceedings of the 2000 Congress on Evolutionary Computation, LA Jolla, CA, USA, 16-19 July 2000; pp. 256-263.

56. Park, M.G.; Lee, M.C. Artificial potential field based path planning for mobile robots using a virtual obstacle concept. In Proceedings of the 2003 IEEE/ ASME International Conference on Advanced Intelligent Mechatronics, Kobe, Japan, 20-24 July 2003; pp. 735-740.

57. Cao, Q.X.; Huang, Y.W.; Zhou, J.L. An evolutionary artificial potential field algorithm for dynamic path planning of mobile robot. In Proceedings of the 2006 IEEE/RSJ International Conference on Intelligent Robots and Systems, Beijing, China, 9-15 October 2006; pp. 3331-3336.

58. Zhang, Q.S.; Chen, D.D.; Chen, T. An obstacle avoidance method of soccer robot based on evolutionary artificial potential field. Energy Procedia 2012, 16, 1792-1798. [CrossRef]

59. Zhou, L.; Li, W. Adaptive Artificial Potential Field Approach for Obstacle Avoidance Path Planning. In Proceedings of the 7th International Symposium on Computational Intelligence and Design, Hangzhou, China, 13-14 December 2014; pp. 429-432.

60. Whitbrook, A.M.; Aickelin, U.; Garibaldi, J.M. Idiotypic immune networks in mobile-robot control. IEEE Trans. Syst. Man Cybern. Part B Cybern. 2007, 37, 1581-1598. [CrossRef]

61. Huq, R.; Mann, G.K.I.; Gosine, R.G. Mobile robot navigation using motor schema and fuzzy context dependent behavior modulation. Appl. Soft Comput. J. 2008, 8, 422-436. [CrossRef]

62. Fernandez-Leon, J.A.; Acosta, G.G.; Mayosky, M.A. Behavioral control through evolutionary neurocontrollers for autonomous mobile robot navigation. Robot. Auton. Syst. 2009, 57, 411-419. [CrossRef]

63. Shi, C.X.; Wang, Y.Q.; Yang, J.Y. A local obstacle avoidance method for mobile robots in partially known environment. Robot. Auton. Syst. 2010, 58, 425-434. [CrossRef]

64. Toibero, J.M.; Roberti, F.; Carelli, R.; Fiorini, P. Switching control approach for stable navigation of mobile robots in unknown environments. Robot. Comput. Integr. Manuf. 2011, 27, 558-568. [CrossRef]

65. Marefat, M.; Britanik, J. Case-based process planning using an object-oriented model representation. Robot. Comput. Integr. Manuf. 1997, 13, 229-251. [CrossRef]

66. Zhang, X.C.; Ji, G.; Shao, G.F.; Wei, J.; Li, Z.S. Research on soccer robot learning based on cases. J. Harbin Inst. Technol. 2004, 36, 905-907.

67. Zhang, P.Y.; Lu, T.S.; Song, L.B. The Case-Based Learning of Mot ion Planning and Its SVR Implementation for Volleyball Robot. J. Shanghai Jiaotong Univ. 2006, 40, 461-465.

68. Weng, M.; Du, Q.Y.; Qu, R.; Cai, Z. A Path Planning Algorithm Based on Typical Case Reasoning. Geomat. Inf. Sci. Wuhan Univ. 2008, 33, 1263-1266. [CrossRef]

69. Zhong, X.Y.; Peng, X.F.; Miao, M.L. Planning of robot paths through environment modelling and adaptive window. J. Huazhong Univ. Sci. Technol. 2010, 38, 107-111.

70. Liu, C.M.; Li, Z.B.; Huang, Z.H.; Zuo, L.; Wu, J.; Xu, X. A reactive navigation method of mobile robots based on LSPI and rolling windows. J. Cent. South Univ. Sci. Technol. 2013, 44, 970-977.

71. Chou, C.C.; Lian, F.L.; Wang, C.C. Characterizing Indoor Environment for Robot Navigation Using Velocity Space Approach with Region Analysis and Look-Ahead Verification. IEEE Trans. Instrum. Meas. 2011, 60, 442-451. [CrossRef]

72. Zhang, H.Y. An improved immune algorithm for simple assembly line balancing problem of type 1. J. Algorithms Comput. Technol. 2017, 11, 317-326. [CrossRef] 
73. Khari, M.; Kumar, P.; Burgos, D.; Crespo, R.G. Optimized test suites for automated testing using different optimization techniques. Soft Comput. 2018. [CrossRef]

74. Arora, S.; Singh, S. An Effective Hybrid Butterfly Optimization Algorithm with Artificial Bee Colony for Numerical Optimization. Int. J. Interact. Multimedia Artif. Intell. 2017, 4, 14-21. [CrossRef] 